\title{
Nordihydroguaiaretic Acid, a Lignan from Larrea tridentata (Creosote Bush), Protects Against American Lifestyle-Induced Obesity Syndrome Diet-Induced Metabolic Dysfunction in Mice ${ }^{[\mathbf{S}}$
}

\author{
Jackie K. W. Chan, ${ }^{1}$ Stefanie Bittner, Alex Bittner, Suman Atwal, ${ }^{2}$ Wen-Jun Shen, \\ Mohammed Inayathullah, Jayakumar Rajada, Mark R. Nicolls, Fredric B. Kraemer, \\ and Salman Azhar
}

\begin{abstract}
Geriatrics Research, Education and Clinical Center, Veterans Affairs Palo Alto Health Care System, Palo Alto, California (J.K.W. C., S.B., A.B., S.At., W.-J.S., F.B.K., S.Az.); and Division of Endocrinology, Gerontology, and Metabolism (J.K.W.C., S.B., A.B., S.At., W.-J.S., F.B.K., S.Az.), BioADD Laboratory, and Divisions of Cardiovascular Pharmacology CVI (M.I., J.R.) and Pulmonary and Critical Care Medicine (M.R.N.), Stanford University, Stanford, California
\end{abstract}

Received August 23, 2017; accepted February 16, 2018

\begin{abstract}
To determine the effects of nordihydroguaiaretic acid (NDGA) on metabolic and molecular changes in response to feeding a typical American fast food or Western diet, mice were fed an American lifestyle-induced obesity syndrome (ALIOS) diet and subjected to metabolic analysis. Male C57BL/6J mice were randomly assigned to the ALIOS diet, the ALIOS diet supplemented with NDGA (NDGA+ALIOS), or a control diet and were maintained on the specific diet for 8 weeks. Mice fed the ALIOS diet showed increased body, liver, and epididymal fat pad weight as well as increased plasma alanine transaminase (ALT) and aspartate aminotransferase (AST) levels (a measure of liver injury) and liver triglyceride content. Coadministration of NDGA normalized body and epididymal fat pad weight, ALT and AST levels, and liver triglycerides. NDGA treatment also improved insulin sensitivity but not glucose intolerance in mice fed the ALIOS diet. In mice fed the NDGA+ALIOS diet, NDGA supplementation induced peroxisome proliferator-activated receptor $\alpha$
\end{abstract}

(PPAR $\alpha$; the master regulator of fatty acid oxidation) and mRNA levels of carnitine palmitoyltransferases Cpt1c and Cpt2, key genes involved in fatty acid oxidation, compared with the ALIOS diet. NDGA significantly reduced liver endoplasmic reticulum (ER) stress response C/EBP homologous protein, compared with chow or the ALIOS diet, and also ameliorated ALIOS diet-induced elevation of apoptosis signaling protein, caspase 3. Likewise, NDGA downregulated the ALIOS diet-induced mRNA levels of Pparg, fatty acid synthase Fasn, and diacylglycerol acyltransferase Dgat2. NDGA treatment of ALIOS-fed mice upregulated the hepatic expression of antioxidant enzymes, glutathione peroxidase 4 , and peroxiredoxin 3 proteins. In conclusion, we provide evidence that NDGA improves metabolic dysregulation by simultaneously modulating the PPAR $\alpha$ transcription factor and key genes involved in fatty acid oxidation, key antioxidant and lipogenic enzymes, and apoptosis and ER stress signaling pathways.

\section{Introduction}

The prevalence of obesity has reached epidemic proportions (Ng et al., 2014; NCD Risk Factor Collaboration, 2016); recent survey data indicate that currently $37.7 \%$ of adults and $17.0 \%$ of children in the United States are obese (Seidell and

This work was supported by the U.S. Department of Veterans Affairs (VA) Biomedical Laboratory Research and Development, and the VA Office of Research and Development Merit Review Award Program, and the National Institutes of Health National Heart, Lung, and Blood Institute [Grant 1R01HL92473].

${ }^{1}$ Current affiliation: Abcam, Burlingame, California.

${ }^{2}$ Current affiliation: Carmot Therapeutics Inc., San Francisco, California. https://doi.org/10.1124/jpet.117.243733.

S This article has supplemental material available at jpet.aspetjournals.org.
Halberstadt, 2016). Increasing per capita food supplies and consumption of sugar-sweetened soft drinks, energy-dense food, overnutrition, and sedentary lifestyles (e.g., a reduction in physical activities, excessive television watching, and use of electronic devices) are major contributors to obesity and its associated metabolic complications (Malik et al., 2013; Popkin and Hawkes, 2016; Heymsfield and Wadden, 2017). Obesity has been implicated as one of the major risk factors for many chronic metabolic diseases such as type 2 diabetes (T2D) (Kramer et al., 2010; Day and Bailey, 2011; American Diabetes Association, 2016), metabolic syndrome (MetS) (Grundy, 2004; Andersen and Fernandez, 2013), nonalcoholic fatty liver disease (NAFLD) (Fabbrini et al., 2010; Patell et al., 2014), certain cancers (Deng et al., 2016), and cardiovascular

ABBREVIATIONS: ALIOS, American lifestyle-induced obesity syndrome; ALT, alanine transaminase; AST, aspartate aminotransferase; AUC, area under the curve; CAT, catalase; CHOP, C/EBP homologous protein; CPT, carnitine palmitoyltransferase; CVD, cardiovascular disease; ER, endoplasmic reticulum; GPX, glutathione peroxidase; HOMA-IR, homeostatic model assessment-estimated insulin resistance; MetS, metabolic syndrome; NAFLD, nonalcoholic fatty liver disease; NDGA, nordihydroguaiaretic acid; NDGA+ALIOS, American lifestyle-induced obesity syndrome diet supplemented with nordihydroguaiaretic acid; NEFA, nonessential fatty acid; PPAR, peroxisome proliferator-activated receptor; SOD, superoxide dismutase; T2D, type 2 diabetes. 
disease (CVD) (Zalesin et al., 2011; Bastien et al., 2014). The prevalence of these clinical conditions is also increasing at an accelerated pace. In addition, both MetS and NAFLD independently increase the risk of T2D and CVD (Grundy, 2006; Haffner, 2006; Smith and Adams, 2011; Anstee et al., 2013). In contrast to T2D and CVD, however, currently no pharmacological agents are specifically approved for the treatment of NAFLD, including nonalcoholic steatohepatitis (Chalasani et al., 2012; Dyson et al., 2014; Barb et al., 2016) and MetS (Swislocki et al., 2012; Lim and Eckel, 2014).

Previous studies from our laboratory have shown that nordihydroguaiaretic acid (NDGA), one of the major constituents of Creosote bush, Larrea tridentata, exerts profound effects on a number of metabolic abnormalities, including improving obesity, dyslipidemia, insulin resistance, hypertension, steatosis, and altered glucose metabolism, in several rodent models (Zhang et al., 2013, 2015, 2016). Although these studies provided important mechanistic information by which NDGA improves metabolic abnormalities, they were carried out mainly using genetically manipulated (e.g., $o b / o b$ mice) animals fed an abnormally high-carbohydrate (high-fructose) or high-fat diet; none of these models fully reproduces the major pathophysiological changes associated with the consumption of typical American fast food or a "Western" diet (Tetri et al., 2008). The goal of our studies was to examine the effect of NDGA administration on metabolic dysfunction in a previously described nutritionally induced mouse model of dyslipidemia and insulin resistance, which was developed using dietary formulation based on commonly consumed fast foods (i.e., mice are fed a high-fat chow containing trans fats and are given the required amount of high-fructose corn syrup equivalents) (Tetri et al., 2008). This model is commonly referred to as the American lifestyle-induced obesity syndrome (ALIOS) model. We focused our studies on hepatic metabolism, considering that the liver is the major site for intermediary metabolism of carbohydrates, fats, and proteins. The liver is also a major storage site of carbohydrate (glycogen) and lipids (cholesterol/cholesteryl esters and triglycerides). Here, we provide evidence that dietary administration of NDGA to ALIOS mice improves metabolic dysregulation by upregulation of peroxisome proliferator-activated receptor $\alpha$ $(\operatorname{PPAR} \alpha)$ protein, increased expression of key genes involved in fatty acid oxidation, and selected antioxidant enzymes and simultaneously downregulation of the mRNA expression of key lipogenic enzymes, apoptosis, and endoplasmic reticulum (ER) stress signaling pathways.

\section{Materials and Methods}

Antibodies. Anti- $\beta$-actin, anti-caspase 3 , and anti-catalase (CAT) antibodies were purchased from Cell Signaling Technology (Danvers, MA). Anti-glutathione peroxidase 1 (GPX1), anti-PPAR $\alpha$, anti-PPAR $\gamma$, anti-GPX4, and anti-superoxide dismutase 2 (SOD2)/ MnSOD antibodies were supplied by Abcam (Cambridge, MA). Anti$\mathrm{C} / \mathrm{EBP}$ homologous protein (CHOP) antibody was from Proteintech Group Inc. (Rosemont, IL). IRDye680RD goat anti-rabbit IgG $(\mathrm{H}+\mathrm{L}$ ) secondary antibody was purchased from LI-COR Biosciences (Lincoln, NE). Other antibody details are presented in Supplemental Table 1.

Chemicals and Reagents. The Pierce BCA Protein Assay Kit was purchased from Thermo Fisher Scientific (Waltham, MA). NDGA was isolated and purified from Creosote bush (L. tridentata) by Pharmaceutics International Inc. (Hunt Valley, MD) according to a slight modification (Elakovich and Stevens, 1985) of the procedure of
Waller and Gisvold (1945). In brief, dried leaves and small twigs of L. tridentata (collected from the deserts of Arizona) were extracted successively with organic solvents (methyl isobutyl ketone, ethyl ether, acetone, and methanol) followed by water in a Soxhlet extractor. Subsequent purification of the combined concentrated extract by Sephadex column chromatography followed by methanol repurification from the concentrated column eluent containing NDGA yielded beige-cream-colored NDGA powder. The bulk quantity of such a purified preparation of NDGA was supplied by Mr. Glen Kelley (Insmed Inc., Richmond, VA) (Kelley et al., 2004). The purity of NDGA was $>97 \%$ as determined by nuclear magnetic resonance analysis (Supplemental Fig. 1). Reagents for measuring serum alanine transaminase (ALT), aspartate aminotransferase (AST), glucose, triglycerides, and total cholesterol were purchased from Stanbio Laboratory (Boerne, TX). Free cholesterol and nonesterified fatty acid (NEFA) measurement kits were purchased from Wako Diagnostics (Richmond, VA). Humulin R U-100 (insulin human) was supplied by Lilly USA (Indianapolis, IN). Glucose was purchased from Sigma-Aldrich (St. Louis, MO). All other reagents used were of analytical grade. The custom ALIOS diet (no. TD06303) supplemented with NDGA (NDGA+ALIOS) or without NDGA (2.5 g/kg diet) was prepared by Harlan Teklad/Envigo (Madison, WI).

Animal Studies. Six-week-old male C57Bl/6J mice were obtained from the Jackson Laboratory (Bar Harbor, ME). All animal experiments were performed according to procedures approved by the Veterans Affairs Palo Alto Health Care System Animal Care and Use Committee. Animals were housed in laboratory cages at $23^{\circ} \mathrm{C}$ under a 12-hour/12-hour light/dark cycle. Mice were separated into three treatment groups (chow, ALIOS, and NDGA+ALIOS) normalized by body weight and allowed to acclimate for 2 weeks prior to the study. Mean body weight at the start of the study was $24.8 \pm 0.3 \mathrm{~g}$. Mice were housed socially, four per cage, and were fed either standard chow or the ALIOS diet (Tetri et al., 2008). Briefly, the ALIOS diet is a modified high-fat diet in which $45 \%$ of the caloric content is derived from fat; $30 \%$ of the fat is present in the form of partially hydrogenated vegetable oil, consisting of $28 \%$ saturated, $57 \%$ monounsaturated, and $13 \%$ polyunsaturated fatty acids. In addition, drinking water for the ALIOS groups was supplemented with high-fructose corn syrup equivalents $(55 \%$ fructose, $45 \%$ glucose) at $42 \mathrm{~g} / \mathrm{l}$. Mice fed this combination diet become obese and develop glucose intolerance, hyperinsulinemia, and hepatic steatosis associated with a necroinflammatory and profibrogenic response. For the NDGA+ALIOS group, the ALIOS diet was further augmented with $2.5 \mathrm{~g} / \mathrm{kg}$ diet NDGA. Animals were maintained on these diets for 8 weeks. Body weights and food and water consumption were measured weekly.

Glucose Tolerance Test and Insulin Tolerance Test. The glucose tolerance test (GTT) and the insulin tolerance test (ITT) were performed separately, at least 1 week apart, at weeks 7 and 8 of the study, respectively. For the GTT, animals were fasted for 16 hours overnight, and glucose ( $1 \mathrm{~g} / \mathrm{kg}$ body weight) was injected intraperitoneally. Blood was collected from the tail vein prior to injection and at $15,30,60$, and 120 minutes postinjection to assess glucose clearance. For ITT, animals underwent a 6 -hour fast. Insulin $(0.75 \mathrm{U} / \mathrm{kg})$ was injected intraperitoneally and blood was collected prior to injection and at $15,30,45$, and 60 minutes postinjection to assess insulin sensitivity. Blood glucose levels were determined immediately using a glucometer (Precision Xtra; Abbott, Abbott Park, IL). The total area under the curve (AUC) was calculated using the trapezoidal method.

Quantification of Serum Metabolites. Blood was collected from the tail vein after a 4-hour fast at the final week of the experimental period. Samples were centrifuged at $4000 \mathrm{~g}$ for 15 minutes at $4^{\circ} \mathrm{C}$ and serum was retained and stored at $-80^{\circ} \mathrm{C}$. Serum ALT, AST, glucose, triglyceride, total cholesterol, free cholesterol, and NEFA levels were determined with commercial assays according to the manufacturers' protocols.

Measurement of Liver Triglyceride Content. Suitable aliquots (in duplicate) of liver homogenates were extracted with a mixture of chloroform and methanol according to the procedure of 
Folch et al. (1957), and lipid samples were subsequently quantified for their triglyceride content using an enzymatic colorimetric assay kit supplied by Stanbio Laboratory as described previously (Zhang et al., 2013).

RNA Isolation and Quantitative Real-Time Polymerase Chain Reaction. Total hepatic RNA was isolated using the Qiagen RNeasy Mini Kit (Qiagen, Valencia, CA) according to the manufacturer's instructions. RNA purity was confirmed using spectrophotometric absorbance at $260 / 280 \mathrm{~nm}$, and $2 \mu \mathrm{g}$ total RNA was reverse transcribed to cDNA using a High-Capacity RNA-to-cDNA kit (Thermo Fisher Scientific). Primers were designed from PrimerBLAST (http://www.ncbi.nlm.nih.gov/tools/primer-blast/) and are listed in Table 1. Quantitative real-time polymerase chain reaction reactions were amplified using a FastStart Universal SYBR Green Master PCR Kit (Roche, Mannheim, Germany) on an ABI Prism 7700 system (Applied Biosystems, Foster City, CA). Results were calculated using the comparative threshold cycle method (Livak and Schmittgen, 2001) using acidic ribosomal phosphoprotein, large, P0 (36B4 or ribosomal protein lateral stalk subunit P0) as the reference gene. Results are expressed as a fold change in gene expression relative to chow-fed animals, normalized to 1.0.

Western Blot Analysis. Flash-frozen liver tissue was homogenized in radioimmunoprecipitation assay lysis buffer (Santa Cruz Biotechnology, Dallas, TX) and protein concentrations were determined with BCA Protein Reagent (Pierce Biotechnology, Rockford, IL). Samples were reduced for SDS-PAGE application, and 20-40 $\mu \mathrm{g}$ protein per lane was electrophoresed on $12 \%$ Bis-Tris polyacrylamide gels (Bio-Rad, Hercules, CA) and subsequently transferred to nitrocellulose membranes (GE Healthcare, Little Chalfont, UK). Membranes were blocked with $5 \%$ bovine serum albumin in Tris-buffered saline with $0.1 \%$ Tween 20 and incubated with each of the following antibodies: rabbit anti-PPAR $\alpha$, rabbit anti-CHOP, rabbit anti-caspase 3, rabbit anti-PPAR $\gamma$, rabbit anti-CAT, rabbit antiGPX1, rabbit anti-GPX4, rabbit anti-SOD2/MnSOD, and rabbit anti- $\beta$-actin. Subsequently, membranes were incubated with IRDye 680LT goat anti-rabbit secondary antibody (LI-COR Biosciences) and protein bands were visualized and quantified with the Odyssey Infrared Imaging System (LI-COR Biosciences).

Statistical Analysis. Data are reported as the mean \pm S.E.M. Comparisons were made using one- and two-way analyses of variance with Bonferroni's multiple-comparisons test using GraphPad Prism 6.0 software (GraphPad Inc., La Jolla, CA). $P$ values of $<0.05$ were considered statistically significant.

\section{Results}

To investigate the effects of the ALIOS diet consumption, we measured several physiologic parameters during the 8-week study. Terminal body weights of mice fed chow weighed an average of $31 \mathrm{~g}$. Animals fed the ALIOS diet were significantly heavier, averaging $34 \mathrm{~g}(P=0.0007)$. NDGA supplementation in the ALIOS diet attenuated weight gain and was comparable to chow at $32 \mathrm{~g}$ (Fig. 1A). Liver and epididymal fat weights were also significantly increased in the ALIOS group; however, when normalized by body weight, only epididymal fat was significant (Fig. 1B). Mice fed a chow diet had livers weighing $1.05 \mathrm{~g}$ compared with ALIOS-fed mice (1.31 g; $P=$ 0.0001 ), whereas liver weights were $1.13 \mathrm{~g}$ for NDGA+ALIOSfed mice $(P=0.0330$ compared with ALIOS). There was no significant difference between the chow and NDGA+ALIOS groups $(P=0.8252)$. Linear regression on growth curves demonstrated a similar trend comparing the three groups. Although the chow and NDGA+ALIOS groups, on average, gained approximately 0.10 and $0.11 \mathrm{~g}$ daily, respectively, ALIOS weight gain was substantially greater at $0.14 \mathrm{~g} / \mathrm{day}$ (Fig. 1C). However, the increased weight gain in the ALIOS group was not attributable to increased diet consumption. Although chow-fed animals ate the most diet in terms of mass, the ALIOS and NDGA+ALIOS groups had similar intake (Fig. 1D). When diet consumption was converted to total caloric intake, the ALIOS and NDGA+ALIOS groups ingested the most daily (approximately $27 \%$ and $20 \%$ more than chowfed animals, respectively) (Fig. 1E).

Next, because we observed significant weight gain in the ALIOS-fed group, we assessed the impact of the diet on glucose tolerance and insulin sensitivity by performing the GTT and ITT, respectively. After an overnight fast, basal glucose levels were similar among the three groups, at 70, 78, and $78 \mathrm{mg} / \mathrm{dl}$ for the chow, ALIOS, and NDGA+ALIOS groups, respectively. Tail vein glucose readings reached maximal levels at 30 minutes after a $1-\mathrm{g} / \mathrm{kg}$ dose of glucose; chow-fed animals averaged a peak of $271 \mathrm{mg} / \mathrm{dl}$. In comparison, the ALIOS and NDGA+ALIOS groups spiked significantly higher, at $324(P<0.0001)$ and $309 \mathrm{mg} / \mathrm{dl}(P=0.014)$, respectively. There were no differences between the ALIOS and NDGA+ALIOS groups at any time point (Fig. 2A). Calculated total AUCs demonstrated a similar trend; the ALIOS and NDGA+ ALIOS groups had significantly higher AUCs compared with chow-fed animals (Fig. 2B). Insulin tolerance was measured after a week of recovery. Six-hour fasted glucose levels indicated significant differences between the three dietary treatment groups. Chow-fed animals had the lowest fasting glucose at $141 \mathrm{mg} / \mathrm{dl}$. Whereas the NDGA+ ALIOS group averaged serum glucose levels at $172 \mathrm{mg} / \mathrm{dl}$, serum levels of animals solely fed the ALIOS diet were further elevated at $210 \mathrm{mg} / \mathrm{dl}(P=0.0006)$. At a $0.75-\mathrm{U} / \mathrm{kg}$ insulin dose, serum glucose levels were decreased to 47,84 , and $56 \mathrm{mg} / \mathrm{dl}$ at 60 minutes postinjection in the chow, ALIOS, and NDGA+ALIOS groups, respectively. Serum glucose was significantly elevated in the ALIOS group at every time point, whereas the insulin tolerance profile in the NDGA+ALIOS

TABLE 1

Mouse primers used for quantitative real-time polymerase chain reaction Gene symbols are listed in italics.

\begin{tabular}{|c|c|c|}
\hline Gene Name & Forward Primer & Reverse Primer \\
\hline CPT1a $(C p t 1 a)$ & 5'-CAGACTCGGTCACCACTCAA-3' & 5'-TTGAGGGCTTCATGGCTCAG-3' \\
\hline CPT1c $(C p t 1 c)$ & 5'-CAGATCCAGCCGTCCATCTC-3' & 5'-TGCAGGACCAACTGGATGAA-3' \\
\hline CPT2 (Cpt2) & 5'-GCACAGAGACTATTCGCCCA-3' & 5'-GGTCAGCTGGCCATGGTATT-3' \\
\hline DGAT2 (Dgat2) & $5^{\prime}$-ACTGGAACACGCCCAAGAAA-3' & $5^{\prime}$-GTAGTCTCGGAAGTAGCGCC-3' \\
\hline FASN (Fasn) & 5'-CTGTGCCCGTCGTCTATACC-3' & 5'-AACCTGAGTGGATGAGCACG-3' \\
\hline $\operatorname{PPAR} \alpha$ (Ppara) & 5'-ACAGGAGAGCAGGGATTTGC-3' & 5'-TACCTACGCTCAGCCCTCTT-3' \\
\hline 36B4 (Rplp0) & 5'-TTTGGGCATCACCACGAAAA-3' & 5'-GGACACCCTCCAGAAAGCGA-3' \\
\hline
\end{tabular}

$R p l p 0$, ribosomal protein lateral stalk subunit $\mathrm{P} 0$. 

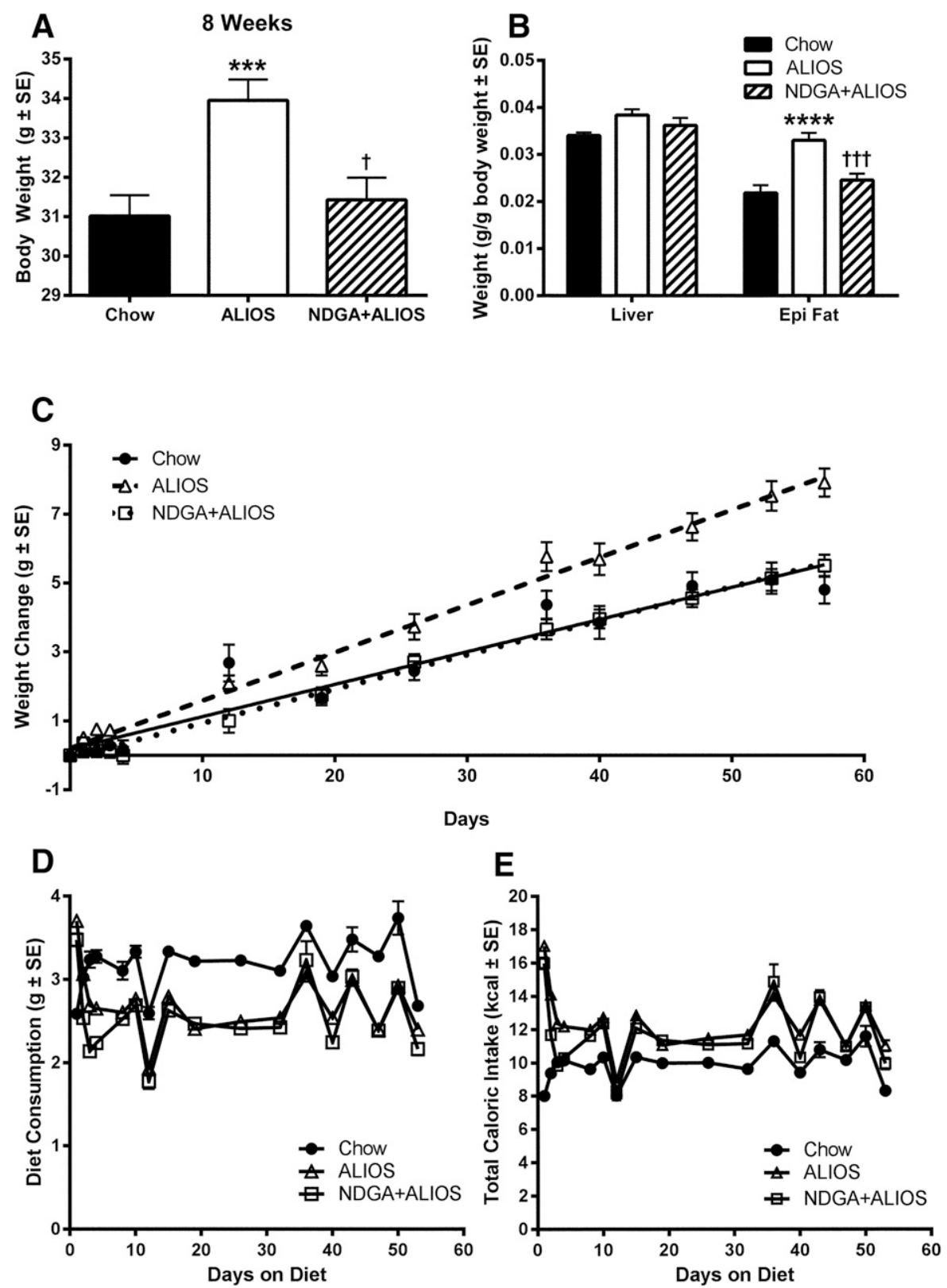

Fig. 1. Changes in metabolic parameters after 8 weeks of chow, ALIOS, or NDGA+ALIOS diet consumption. (A) Changes in terminal body weights. (B) Changes in liver and epididymal fat weights. (C) Time-dependent changes in the body weight of mice fed the chow, ALIOS, or NDGA+ALIOS diet. (D) Diet consumption. (E) Total calorie intake. Mice were housed socially, four per cage. (A) Chow, $\mathrm{n}=16$, ALIOS, $\mathrm{n}=16$, and NDGA+ALIOS, $n=8$ (ANOVA). (B) Chow, $n$ $=16$, ALIOS, $\mathrm{n}=16$, and NDGA+ALIOS, $\mathrm{n}=$ 8 (two-way ANOVA). (C-E) Chow, $\mathrm{n}=8$, ALIOS, $\mathrm{n}=8$, and NDGA+ALIOS, $\mathrm{n}=8$ (two-way ANOVA). $* * * P<0.001$ vs. chow; $* * * * P<$ 0.0001 vs. chow; ${ }^{\dagger \dagger} P<0.001$ vs. ALIOS. ANOVA, analysis of variance.

group was similar compared with the chow group (Fig. 2C). Although calculated total AUC values were higher in the NDGA+ALIOS group compared with the chow group, NDGA supplementation significantly decreased the AUC compared with the ALIOS group only $(P=0.0023)$ (Fig. 2D). Four-hour fasted serum glucose and insulin levels were measured at 4 weeks. Glucose levels were increased significantly in ALIOSfed mice $(225 \mathrm{mg} / \mathrm{dl})$ compared with chow-fed mice $(186 \mathrm{mg} / \mathrm{dl})$ $(P=0.04)$, whereas NDGA+ALIOS-fed mice had decreased glucose levels $(189 \mathrm{mg} / \mathrm{dl})(P=0.06)$. The ALIOS diet did not significantly change insulin levels $(0.586 \mathrm{ng} / \mathrm{dl}$ vs. $0.610 \mathrm{ng} / \mathrm{dl})$, but insulin decreased with NDGA $(0.330 \mathrm{ng} / \mathrm{ml}, P=0.001)$. Likewise, the homeostatic model assessment-estimated insulin resistance (HOMA-IR) was not changed by the ALIOS diet (8.338), but NDGA-treated mice had significantly lower HOMA-IR (3.738, $P=0.0008)$. NDGA-treated mice had significantly lower HOMA- $\beta$ (24.63) compared with chow-fed mice (44.14) $(P<0.0001)$, although there was no significant difference compared with ALIOS-fed mice (35.63).

To assess liver injury, we measured serum ALT and AST activity levels in response to feeding mice the ALIOS diet. As shown in Fig. 3, A and B, serum ALT and AST activity levels were significantly elevated after ALIOS diet consumption compared with chow $(P=0.0078$ and $P=0.0199$, respectively). NDGA supplementation lowered ALT and AST levels to values statistically insignificantly different from chow $(P=$ 0.1276 and $P=0.1417$, respectively). Serum triglyceride, glucose, cholesterol, free cholesterol, and NEFA levels were also quantified. Animals fed the ALIOS diet had significantly reduced triglyceride levels $(P<0.0001)$ that were further reduced after NDGA supplementation (Fig. 3C). Triglyceride content was also measured in the liver. As expected, ALIOSfed animals had significantly elevated liver triglycerides $(P=$ 0.0196). Whereas NDGA supplementation ameliorated this 


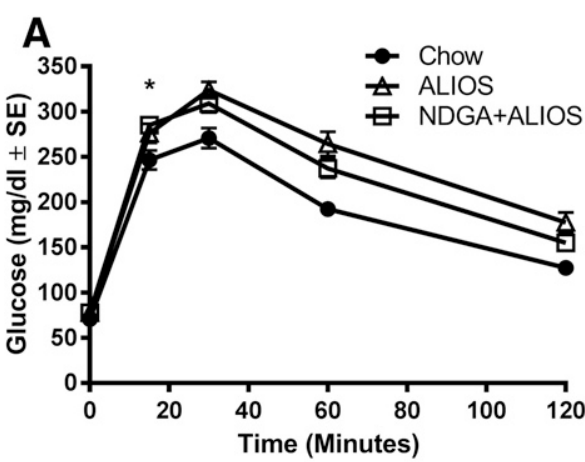

C

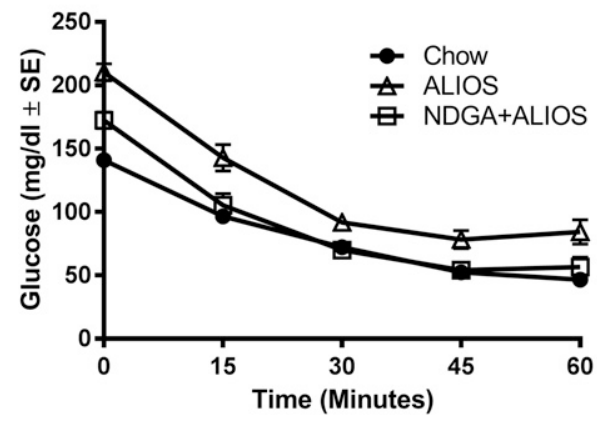

B

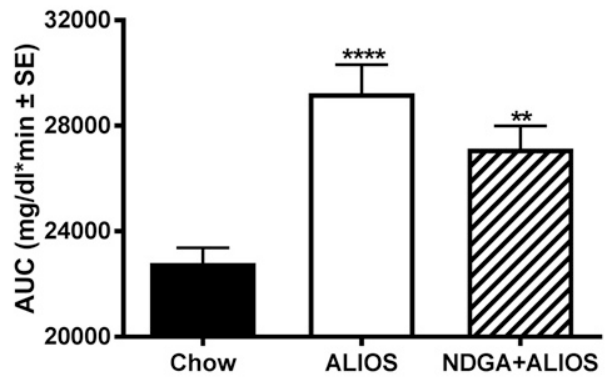

D

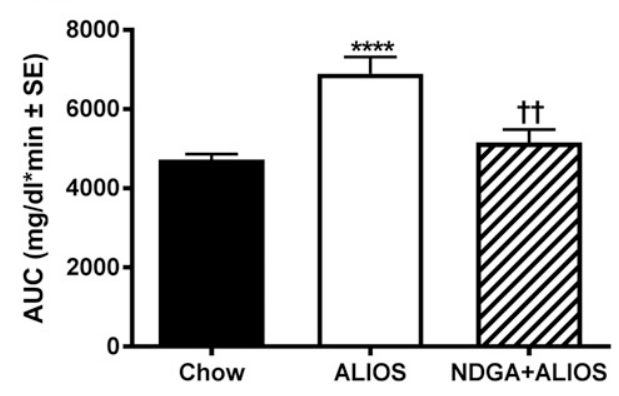

Fig. 2. Glucose and insulin tolerance tests. (A) The GTT demonstrated glucose intolerance in the ALIOS and NDGA +ALIOS groups after 8 weeks. (B) The total AUC of the GTT (in milligrams per deciliter * minute) was significantly elevated in the ALIOS and NDGA+ALIOS groups. (C) The ITT showed a substantially higher curve in the ALIOS group, whereas the chow and NDGA+ALIOS groups remained comparable. (D) The total AUC of the ITT (in milligrams per deciliter $*$ minute) revealed significant elevation in the ALIOS group compared with both the chow and NDGA+ALIOS groups. The total AUC was calculated using the trapezoidal method. Chow, $\mathrm{n}=$ 23, ALIOS, $\mathrm{n}=24$, and NDGA+ALIOS, $\mathrm{n}$ $=16$. $(\mathrm{A}$ and $\mathrm{C}$ ) Two-way ANOVA. (B and D) ANOVA. $* * P<0.01$ vs. chow; $* * * * P<$ 0.0001 vs. chow; ${ }^{\dagger} P<0.01$ vs. ALIOS. ANOVA, analysis of variance. effect, levels between animals fed chow or the NDGA+ALIOS diet, however, were comparable (Fig. 3D). Furthermore, other serum tests demonstrated increased glucose and cholesterol but decreased NEFAs (Table 2) in ALIOS-fed mice. NDGA supplementation did not affect these values.
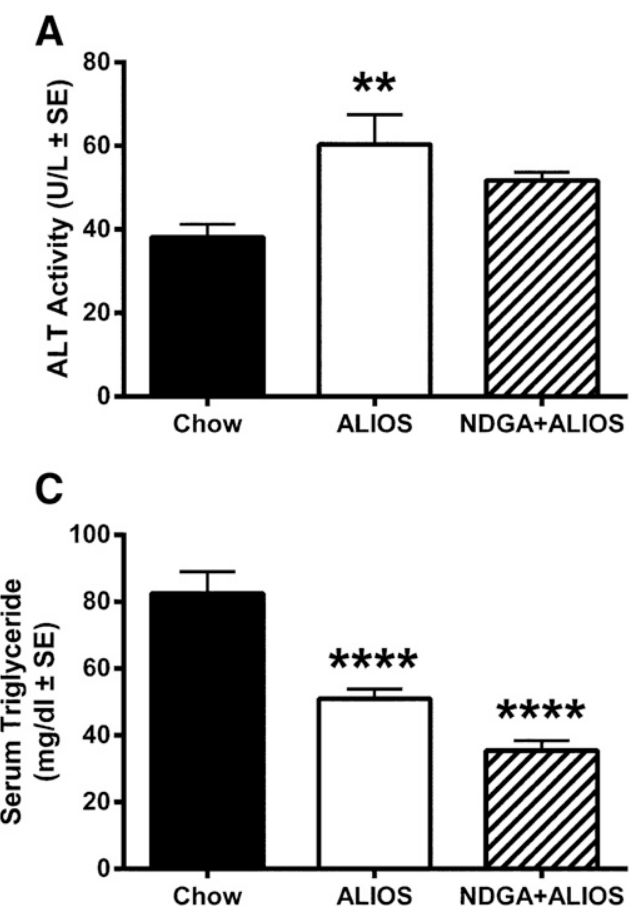

Next, key proteins of several pathways were measured to explore potential molecular mechanisms responsible for the altered liver triglyceride content after NDGA supplementation. Since NDGA had been shown previously to induce the $\operatorname{PPAR} \alpha$ pathway, we measured Ppar $\alpha$ expression in the liver
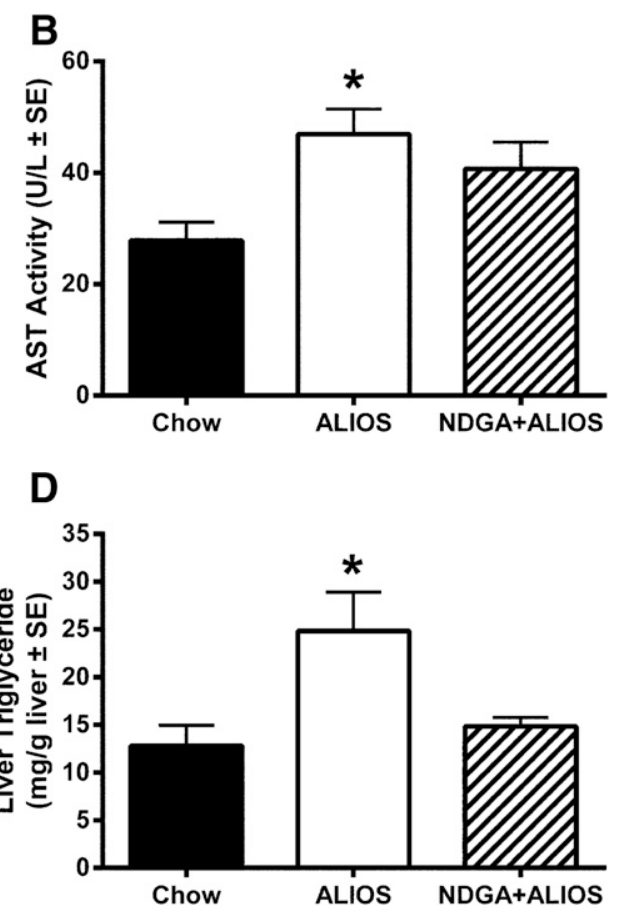

Fig. 3. Liver function tests. (A) ALT was significantly elevated in the ALIOS and NDGA+ALIOS groups compared with animals in the chow group. (B) AST was increased in the ALIOS and NDGA+ALIOS groups. (C) Serum triglycerides were drastically reduced in the ALIOS and NDGA+ALIOS groups. (D) Liver triglycerides were substantially elevated in the ALIOS-fed animals. Levels in the chow and NDGA+ALIOS groups were comparable. (A and B) Chow, $\mathrm{n}=7$, ALIOS, $\mathrm{n}=7$, and NDGA+ALIOS, $\mathrm{n}=8$ (ANOVA). (C) Chow, $\mathrm{n}=15, \mathrm{ALIOS}, \mathrm{n}=16$, and NDGA+ALIOS, $\mathrm{n}=8$ (ANOVA). (D) Chow, $\mathrm{n}=16$, ALIOS, $\mathrm{n}=16$, and NDGA+ALIOS, $\mathrm{n}=8$ (ANOVA). $* P<0.05$ vs. chow; $* * P<0.01$ vs. chow; $* * * * P<0.0001$ vs. chow. ANOVA, analysis of variance. 
TABLE 2

Serum metabolites

Results are expressed as the mean \pm S.E.

\begin{tabular}{lccc}
\hline \multicolumn{1}{c}{ Metabolite } & Chow $(n=15)$ & ALIOS $(n=16)$ & NDGA+ALIOS $(n=8)$ \\
\hline Glucose $(\mathrm{mg} / \mathrm{dl})$ & $186.59 \pm 8.25$ & $230.52 \pm 8.35^{*}$ & $231.29 \pm 8.38^{*}$ \\
Total cholesterol $(\mathrm{mg} / \mathrm{dl})$ & $110.01 \pm 3.26$ & $134.82 \pm 7.01^{* *}$ & $144.40 \pm 7.82^{*}$ \\
Free cholesterol $(\mathrm{mg} / \mathrm{dl})$ & $40.19 \pm 2.24$ & $43.82 \pm 2.48$ & $42.73 \pm 1.50$ \\
NEFA $(\mathrm{mEq} / \mathrm{l})$ & $1.16 \pm 0.19$ & $0.66 \pm 0.05^{* *}$ & $0.49 \pm 0.02^{*}$ \\
\hline
\end{tabular}

$* P<0.01$ vs. chow; ${ }^{*} P<0.05$ vs. chow (analysis of variance).

(Fig. 4A). Compared with chow, Ppar $\alpha$ mRNA was unaffected by the ALIOS diet. NDGA supplementation induced Ppar $\alpha$ 4-fold; however, due to the large variance within the group, this association was statistically insignificant $(P=0.078)$.
NDGA significantly induced $\operatorname{PPAR} \alpha$ protein $>6$-fold compared with the chow $(P=0.0012)$ and ALIOS $(P=0.0113)$ groups. ER stress response and apoptosis signaling proteins CHOP and caspase-3 were measured. NDGA significantly
A Chow

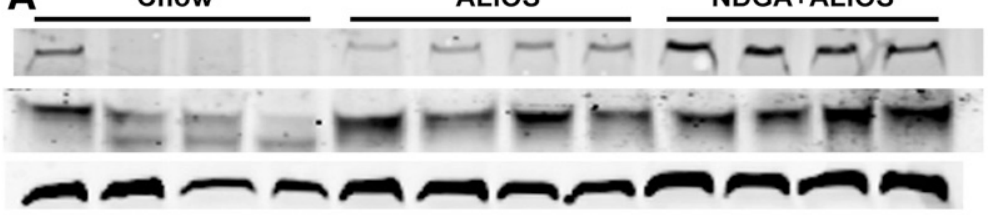

PPAR $\alpha$ qPCR
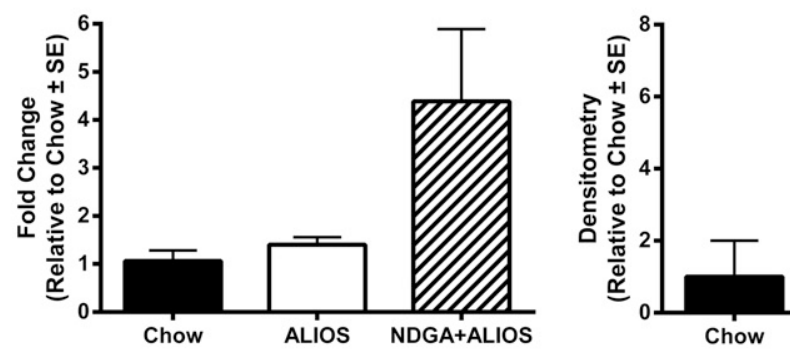

PPAR $\alpha$ WB

CASP3 WB
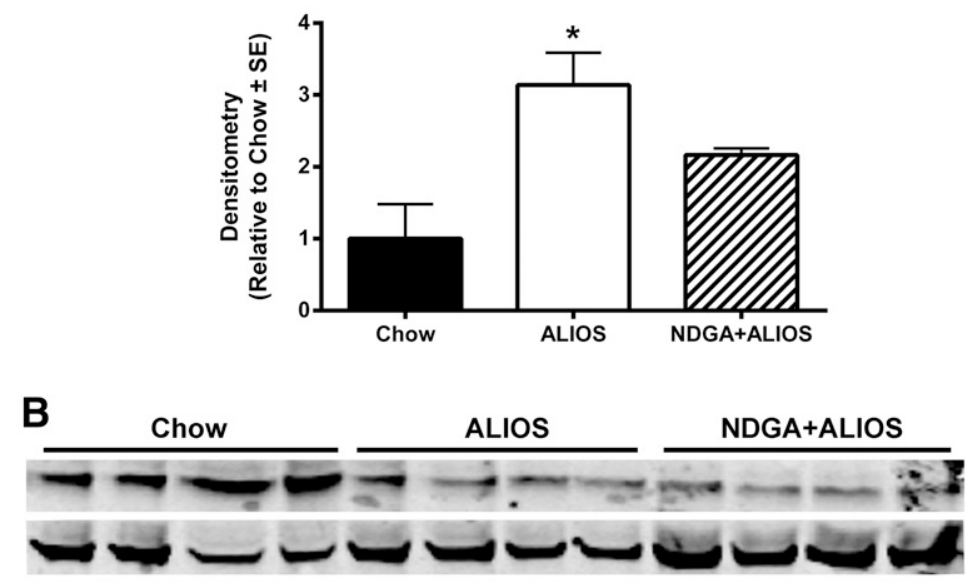

CHOP WB

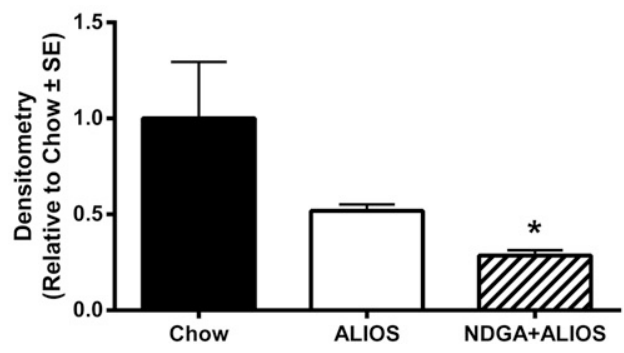

PPAR $\alpha$

CASP3

$\beta$-Actin

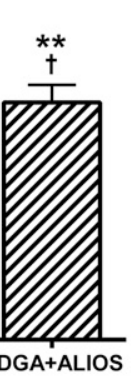

Fig. 4. NDGA modulation of PPAR $\alpha$ expression and ER stress response. (A) PPAR $\alpha$ expression was measured in the liver by quantitative real-time polymerase chain reaction and Western blotting. Both techniques revealed NDGA-induced PPAR $\alpha$ upregulation. (B) NDGA significantly reduced $\mathrm{CHOP}$ expression NDGA modestly reduced caspase-3 (CASP3) compared with ALIOS-fed animals. (A) Chow, $\mathrm{n}=4$, ALIOS, $\mathrm{n}=4$, and NDGA+ALIOS, $\mathrm{n}=4$ (analysis of variance). $* P<0.05$ vs. chow; $* * P<$ 0.01 vs. chow; ${ }^{\dagger} P<0.05$ vs. ALIOS. qPCR, quantitative real-time polymerase chain reaction; WB, Western blot.
CHOP

$\beta$-Actin 
reduced liver CHOP protein, compared with chow $(P=0.0495)$ (Fig. 4B), and also ameliorated ALIOS-induced CASP3 elevation (Fig. 4A).

Next, markers from the carnitine palmitoyltransferase (CPT) family, responsible for the $\beta$-oxidation of fatty acids, were analyzed. Cpt1a mRNA level was unaffected by the ALIOS diet and/or NDGA supplementation. However, Cpt1c was significantly induced $>10$-fold with NDGA treatment, compared with both the chow $(P=0.0005)$ and ALIOS $(P=$ 0.0008 ) groups. $C p t 2$ expression was also moderately elevated with NDGA, but not significantly (Fig. 5A). Since the CPT family of genes was significantly altered with NDGA, we wanted to examine the effect on lipid uptake and adipogenic PPAR $\gamma$. In line with expectations, chow-fed animals exhibited very low basal levels of PPAR $\gamma$ protein. However, those fed the ALIOS diet had variable induction (1.9- to 7.3-fold), averaging an approximately 4-fold increase compared with chow; due to the variance within the data, it did not reach statistical significance $(P=0.125)$. NDGA supplementation reduced PPAR $\gamma$ levels to those similar to chow (Fig. 5B). In view of the upregulation seen in PPAR $\gamma$ protein levels and elevated triglyceride levels, we measured the mRNA expression of diacylglycerol acyltransferase Dgat2 and fatty acid synthase Fasn. The ALIOS diet induced Dgat2 expression significantly (6-fold, $P=0.0359$ ); the addition of NDGA reduced it to basal levels. A similar trend was seen for Fasn, but ALIOS-induced elevation was more modest.

Finally, we measured protein level of genes involved in the oxidative stress response, mainly focusing on antioxidant enzymes related to superoxide and hydrogen peroxide metabolism. Expression of antioxidant enzymes did not display a consistent pattern. CAT and GPX1 remained unchanged, whereas GPX4 was selectively induced by NDGA $(P<$ 0.0001) (Fig. 6). SOD2/MnSOD tended to be reduced in the ALIOS group $(P=0.1522)$, but expression levels were not rescued by NDGA supplementation. Finally, the mRNA expression of peroxiredoxin 3, a mitochondrion-specific $\mathrm{H}_{2} \mathrm{O}_{2}$-scavenging antioxidant enzyme, was reduced by feeding the ALIOS diet and was returned to basal levels in response to dietary administration of the ALIOS diet supplemented with NDGA.

\section{Discussion}

In this work, we provide information about the impact of dietary administration of NDGA on ALIOS diet-induced metabolic dysregulation in mice. This diet was developed by Tetri et al. (2008) using a dietary formulation based on commonly consumed fast food; the diet consists of high fat containing trans fats and the required amount of high-fructose corn syrup equivalents given in drinking water. Mice consuming the ALIOS diet developed obesity and metabolic derangements, including hepatic steatosis with liver injury, dyslipidemia, insulin resistance, and glucose intolerance, and exhibited increased expression of key enzyme genes involved in hepatic lipogenesis. Feeding mice an ALIOS diet supplemented with NDGA ameliorated metabolic dysregulation by robustly upregulating $\operatorname{PPAR} \alpha$ protein expression, the master regulator of hepatic fatty acid oxidation. Thus, NDGA increased key PPAR $\alpha$-regulated genes involved in hepatic fatty acid oxidation, secondarily downregulated the
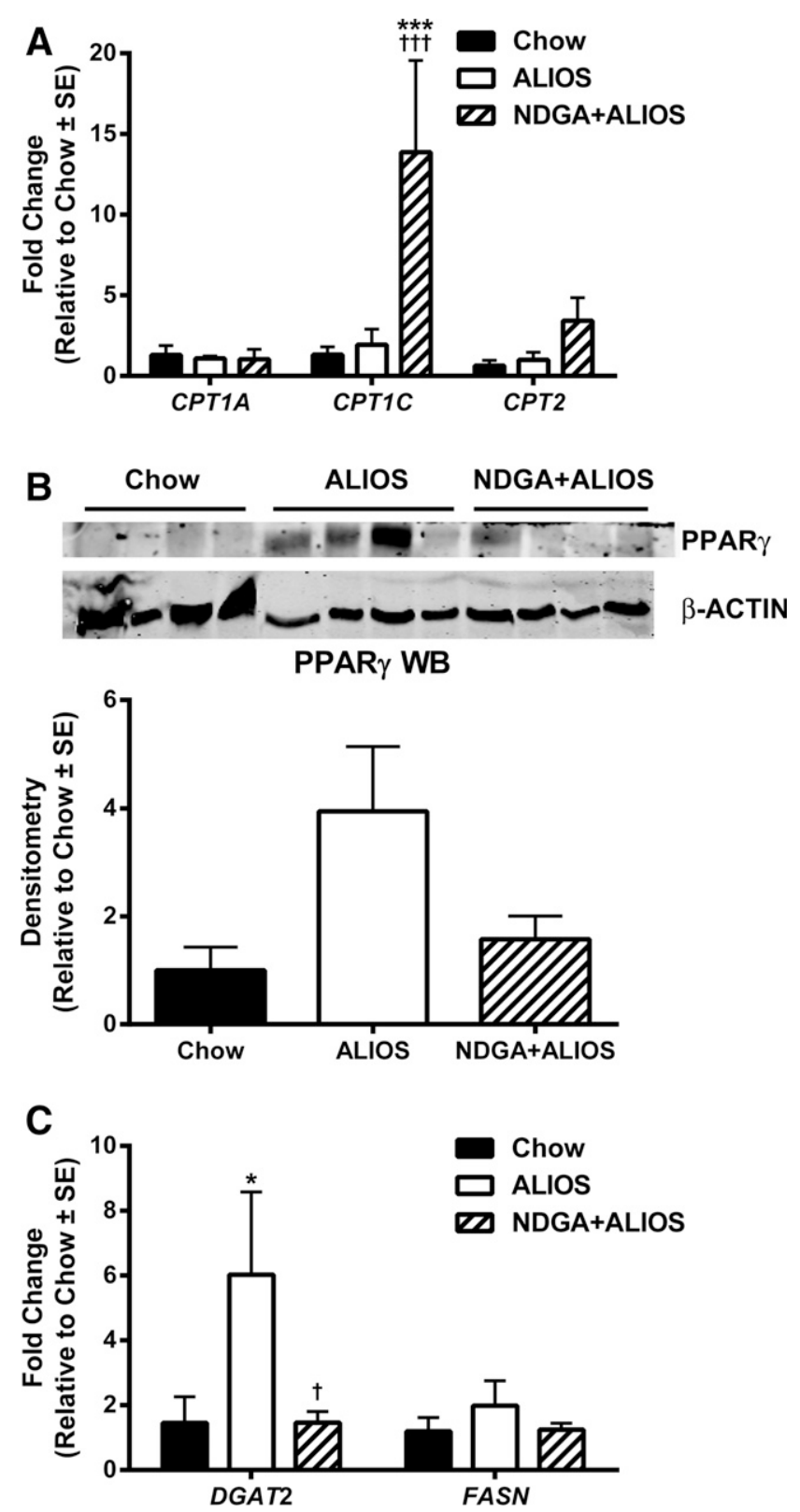

Fig. 5. Effect of NDGA and ALIOS diet on hepatic fatty acid oxidation and adipogenic PPAR $\gamma$. (A) NDGA induced fatty acid oxidation through upregulating Cpt family genes, specifically $C p t 1 c$ and $C p t 2$. (B) The ALIOS diet induced PPAR $\gamma$ protein expression but was suppressed in conjunction with NDGA supplementation. (C) The mRNA levels of triglyceride-forming enzymes diacylglycerol acyltransferase (Dgat2) and fatty acid synthase (Fasn) were both moderately induced after consumption of ALIOS diet. NDGA supplementation prevented such induction. (A and C) Chow, $n=4$, ALIOS, $n=4$, and NDGA+ALIOS, $n=4$ (two-way ANOVA). (B) Chow, $\mathrm{n}=3$, ALIOS, $\mathrm{n}=4$, and NDGA+ALIOS, $\mathrm{n}=$ 4 (ANOVA). $* P<0.05$ vs. chow; $* * * P<0.001$ vs. chow; ${ }^{\dagger \dagger} P<0.001$ vs. ALIOS. ANOVA, analysis of variance; WB, Western blot.

expression of lipogenic genes, markers of ER stress and apoptosis, as well as increased expression of major antioxidant enzymes.

By feeding the ALIOS diet alone for 8 weeks, mice became insulin resistant and glucose intolerant. However, feeding mice the ALIOS diet supplemented with NDGA improved insulin sensitivity, but mice remained glucose intolerant. Previously, we demonstrated that feeding Sprague-Dawley 


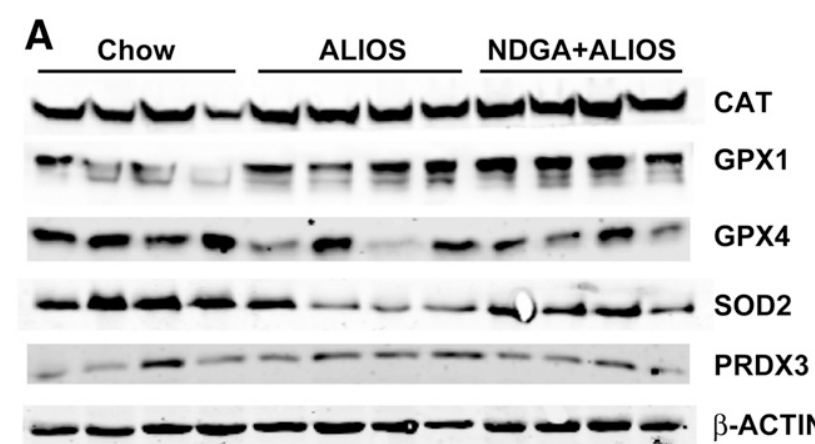

B

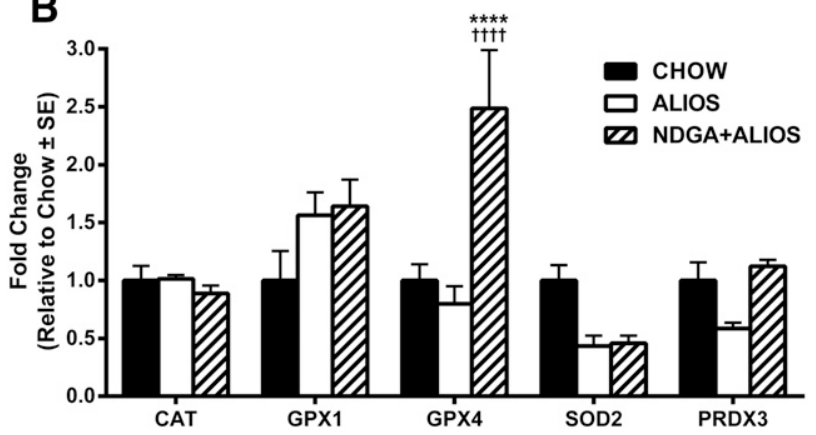

Fig. 6. Impact of NDGA and the ALIOS diet on antioxidant response. (A) A moderate response was observed across multiple antioxidant enzyme proteins. (B) Levels of Gpx4 were significantly elevated in the NDGA +ALIOS group. Levels of SOD2/MnSOD were significantly downregulated after consumption of the ALIOS diet and did not recover in response to simultaneous administration of the ALIOS diet and NDGA. NDGA restored PRDX3 expression to similar levels seen in the chow group. Chow, $\mathrm{n}=4$, ALIOS, $\mathrm{n}=4$, and NDGA+ALIOS, $\mathrm{n}=4$ (two-way analysis of variance). ${ }^{* * * *} P<0.0001$ vs. chow; ${ }^{\dagger \dagger} P<0.0001$ vs. ALIOS. PRDX3, peroxiredoxin 3 .

rats with a high-fructose diet (in which $60 \%$ of total calories were provided as fructose) significantly raised plasma insulin levels (Zhang et al., 2015). A short-term (4 days) NDGA treatment attenuated hyperinsulinemia and fasting plasma glucose and NEFA levels. Analysis of HOMA-IR further confirmed that NDGA treatment ameliorated insulin resistance induced by feeding a high-fructose diet (Zhang et al., 2015). In contrast, we reported previously that NDGA treatment improved both glucose intolerance and insulin sensitivity in $o b / o b$ mice (Zhang et al., 2013). At present, we are unable to provide any explanation as to why NDGA treatment did not improve glucose intolerance in mice maintained on the ALIOS diet. It is possible that the lack of effect of NDGA on ALIOS diet-induced glucose intolerance may be related to the presence of high fructose in the diet. Indeed, high fructose content of the diet is known to induce a wide range of genes along with alterations in both hepatic lipid and carbohydrate metabolism (Koo et al., 2008, 2009; Zhang et al., 2016) and, as such, could impact glucose intolerance in a very complex manner. Further studies are needed to elucidate the complex interactions between high-fructose diet-induced gene changes and alterations in hepatic lipid and glucose metabolism and their relevance to fructose-induced glucose intolerance.

Although the ALIOS diet had no effect on Ppara gene expression, a master regulator of mitochondrial/peroxisomal fatty acid $\beta$-oxidation genes (Rakhshandehroo et al., 2010), supplementation of the ALIOS diet with NDGA caused a robust induction of $\operatorname{PPAR} \alpha$ protein. Consistent with the increased expression of PPAR $\alpha$ protein, NDGA upregulated the mRNA expression of fatty acid oxidation proteins, $C p t 1 c$ and $C p t 2$, which are target genes of PPAR $\alpha$. Cpt1 and Cpt2, together with carnitine/acylcarnitine translocase, facilitate the transport of long-chain fatty acids into the mitochondrial matrix for their $\beta$-oxidation (Bonnefont et al., 2004). The increased expression of Ppar $\alpha$, along with Cpt1 and Cpt2, suggests an increase in mitochondrial fatty acid degradation and energy consumption. In addition, increased expression of PPAR $\alpha$ observed in the NDGA+ALIOS-treated mice strongly points out the possibility that enhanced fatty acid oxidation via activation of $\operatorname{PPAR} \alpha$ and induction of fatty acid oxidation genes is the major mechanism by which NDGA attenuates hepatic steatosis and improves metabolic dysregulation. Although the exact underlying mechanisms are not apparent, the steady-state mRNA levels of Ppar $\alpha$ were not significantly impacted by NDGA treatment, but its protein level was robustly induced. One potential mechanism might be that since NDGA serves as a ligand for PPAR $\alpha$ (Zhang et al., 2013), its binding to $\operatorname{PPAR} \alpha$ protein stabilizes the secondary protein structure and shields it from degradation, thus resulting in increased nuclear protein accumulation. Another possibility is that NDGA promotes posttranslational modification(s) of PPAR $\alpha$ protein (Burns and Vanden Heuvel, 2007; Berrabah et al., 2011; Wadosky and Willis, 2012), leading to its stabilization and enhanced accumulation in the nucleus.

We saw no overall changes in the protein expression levels of adipogenic transcription factor, $\operatorname{PPAR} \gamma$, between mice fed the chow diet and mice fed the ALIOS diet due to large experimental variation. However, there was a trend showing that ALIOS diet treatment upregulated the expression of PPAR $\gamma$ protein, which is known to promote hepatic lipogenesis under altered pathophysiological conditions (Rogue et al., 2010). Such a potential increase is supported by previous studies showing enhanced expression of PPAR $\gamma$ in the fatty livers of several animal models of obesity and diabetes, including $o b / o b$ and $d b / d b$ (Memon et al., 2000), A-Zip (Gavrilova et al., 2003), and KKAy mice (Bedoucha et al., 2001), as well as in the livers of obese patients (Pettinelli and Videla, 2011). Similar to PPAR $\gamma$, the ALIOS diet also induced mRNA expression of key lipogenic genes such as Fasn and Dgat2. Interestingly, coadministration of the ALIOS diet and NDGA significantly reduced the expression of Pparg and two lipogenesis genes. These data suggest that NDGA also ameliorates dyslipidemia by inhibiting $\operatorname{PPAR} \gamma$-induced hepatic lipogenesis. Interestingly, we also found that the ALIOS diet elevated apoptosis signaling protein, caspase 3 , without impacting ER stress response protein CHOP levels in the liver. NDGA suppressed liver CHOP protein and also attenuated ALIOS-induced caspase-3 elevation. Speculatively, NDGA suppression of CHOP and caspase-3 proteins suggests that both apoptosis and ER stress signaling pathways may potentially contribute to ALIOS-induced metabolic dysfunction.

Previous studies have shown that feeding a high-fat diet (Tanaka et al., 2008; Feng et al., 2016) or a high-fructose diet (Chess et al., 2008; Francini et al., 2010) to mice is associated with increased oxidative stress and alteration in the levels of antioxidant enzymes. In this study, we observed variable effects of the ALIOS and NDGA+ALIOS diets on the expression of key antioxidant enzymes and proteins. No changes were noted in the expression of CAT and GPX1, although 
GPX4 protein expression was specifically induced in response to NDGA administration. In contrast, expression of mitochondrial SOD2/MnSOD was downregulated in the ALIOS group. However, NDGA supplementation of the ALIOS diet failed to reverse the inhibitory effect of the ALIOS diet on this enzyme. Finally, the mRNA expression of a mitochondrion-specific $\mathrm{H}_{2} \mathrm{O}_{2}$-scavenging antioxidant enzyme, peroxiredoxin 3 , was blunted in response to feeding the ALIOS diet to animals and returned to normal (control) levels after administration of the ALIOS+NDGA diet. These findings suggest that one additional mechanism by which the ALIOS diet induces metabolic derangements is through amplified oxidative stress insult via suppression of the expression of certain key antioxidant enzymes/proteins. This notion is supported by the observation of a reversal of the ALIOS diet effect on antioxidant enzymes by simultaneous dietary administration of NDGA. In line with this notion, it is well established that NDGA functions as a potent antioxidant (Lü et al., 2010).

In conclusion, our study suggests that 8-week feeding of the ALIOS diet to normal mice is associated with obesity-related metabolic derangements and dysregulation of hepatic metabolism. We further provide evidence that dietary administration of NDGA to ALIOS-fed mice improves metabolic dysregulation by upregulating $\operatorname{PPAR} \alpha$ protein, increased expression of key genes involved in fatty acid oxidation, and selected antioxidant enzymes and simultaneously downregulating the mRNA expression of key lipogenic enzymes and apoptosis and ER stress signaling pathways.

\section{Authorship Contributions}

Participated in research design: Chan, S. Bittner, Atwal, Shen, Kraemer, Azhar.

Conducted experiments: Chan, S. Bittner, Atwal, Inayathullah, Rajada.

\section{Performed data analysis: Chan, A. Bittner.}

Wrote or contributed to the writing of the manuscript: Chan, S. Bittner, A. Bittner, Shen, Nicolls, Kraemer, Azhar.

\section{References}

American Diabetes Association (2016) 6. Obesity management for the treatment of type 2 diabetes. Diabetes Care 39(Suppl 1):S47-S51.

Andersen CJ and Fernandez ML (2013) Dietary strategies to reduce metabolic syndrome. Rev Endocr Metab Disord 14:241-254.

Anstee QM, Targher G, and Day CP (2013) Progression of NAFLD to diabetes mellitus, cardiovascular disease or cirrhosis. Nat Rev Gastroenterol Hepatol 10: 330-344.

Barb D, Portillo-Sanchez P, and Cusi K (2016) Pharmacological management of nonalcoholic fatty liver disease. Metabolism 65:1183-1195.

Bastien M, Poirier P, Lemieux I, and Després J-P (2014) Overview of epidemiology and contribution of obesity to cardiovascular disease. Prog Cardiovasc Dis 56: 369-381.

Bedoucha M, Atzpodien E, and Boelsterli UA (2001) Diabetic KKAy mice exhibit increased hepatic PPARgamma1 gene expression and develop hepatic steatosis upon chronic treatment with antidiabetic thiazolidinediones. J Hepatol 35:17-23.

Berrabah W, Aumercier P, Lefebvre P, and Staels B (2011) Control of nuclear receptor activities in metabolism by post-translational modifications. FEBS Lett $\mathbf{5 8 5}$ 1640-1650.

Bonnefont J-P, Djouadi F, Prip-Buus C, Gobin S, Munnich A, and Bastin J (2004) Carnitine palmitoyltransferases 1 and 2: biochemical, molecular and medical aspects. Mol Aspects Med 25:495-520.

Burns KA and Vanden Heuvel JP (2007) Modulation of PPAR activity via phosphorylation. Biochim Biophys Acta 1771:952-960.

Chalasani N, Younossi Z, Lavine JE, Diehl AM, Brunt EM, Cusi K, Charlton M, and Sanyal AJ (2012) The diagnosis and management of non-alcoholic fatty liver disease: practice guideline by the American Association for the Study of Liver Diseases, American College of Gastroenterology, and the American Gastroenterological Association. Hepatology 55:2005-2023.

Chess DJ, Xu W, Khairallah R, O'Shea KM, Kop WJ, Azimzadeh AM, and Stanley WC (2008) The antioxidant tempol attenuates pressure overload-induced cardiac hypertrophy and contractile dysfunction in mice fed a high-fructose diet. Am J Physiol Heart Circ Physiol 295:H2223-H2230.

Day C and Bailey CJ (2011) Obesity in the pathogenesis of type 2 diabetes. $\mathrm{Br} J$ Diabetes Vasc Dis 11:55-61.
Deng T, Lyon CJ, Bergin S, Caligiuri MA, and Hsueh WA (2016) Obesity, inflammation, and cancer. Annu Rev Pathol 11:421-449.

Dyson JK, Anstee QM, and McPherson S (2014) Non-alcoholic fatty liver disease: a practical approach to treatment. Frontline Gastroenterol 5:277-286.

Elakovich SD and Stevens KL (1985) Phytotoxic properties of nordihydroguaiaretic acid, a lignan from Larrea tridentata (Creosote bush). J Chem Ecol 11:27-33.

Fabbrini E, Sullivan S, and Klein S (2010) Obesity and nonalcoholic fatty liver disease: biochemical, metabolic, and clinical implications. Hepatology $\mathbf{5 1}$ $679-689$.

Feng B, Meng R, Huang B, Shen S, Bi Y, and Zhu D (2016) Silymarin alleviates hepatic oxidative stress and protects against metabolic disorders in high-fat dietfed mice. Free Radic Res 50:314-327.

Folch J, Lees M, and Sloane Stanley GH (1957) A simple method for the isolation and purification of total lipides from animal tissues. J Biol Chem 226:497-509.

Francini F, Castro MC, Schinella G, García ME, Maiztegui B, Raschia MA, Gagliardino JJ, and Massa ML (2010) Changes induced by a fructose-rich diet on hepatic metabolism and the antioxidant system. Life Sci 86:965-971.

Gavrilova O, Haluzik M, Matsusue K, Cutson JJ, Johnson L, Dietz KR, Nicol CJ, Vinson C, Gonzalez FJ, and Reitman ML (2003) Liver peroxisome proliferatoractivated receptor $\gamma$ contributes to hepatic steatosis, triglyceride clearance, and regulation of body fat mass. J Biol Chem 278:34268-34276.

Grundy SM (2004) Obesity, metabolic syndrome, and cardiovascular disease. J Clin Endocrinol Metab 89:2595-2600.

Grundy SM (2006) Metabolic syndrome: connecting and reconciling cardiovascular and diabetes worlds. J Am Coll Cardiol 47:1093-1100.

Haffner SM (2006) The metabolic syndrome: inflammation, diabetes mellitus, and cardiovascular disease. Am J Cardiol 97:3A-11A.

Heymsfield SB and Wadden TA (2017) Mechanisms, pathophysiology, and management of obesity. $N$ Engl $J$ Med 376:254-266.

Kelley GL, Allan G, and Azhar S (2004) High dietary fructose induces a hepatic stress response resulting in cholesterol and lipid dysregulation. Endocrinology 145: $548-555$.

Koo H-Y, Miyashita M, Cho BHS, and Nakamura MT (2009) Replacing dietary glucose with fructose increases ChREBP activity and SREBP-1 protein in rat liver nucleus. Biochem Biophys Res Commun 390:285-289.

Koo H-Y, Wallig MA, Chung BH, Nara TY, Cho BH, and Nakamura MT (2008) Dietary fructose induces a wide range of genes with distinct shift in carbohydrate and lipid metabolism in fed and fasted rat liver. Biochim Biophys Acta 1782 341-348.

Kramer H, Cao G, Dugas L, Luke A, Cooper R, and Durazo-Arvizu R (2010) Increasing BMI and waist circumference and prevalence of obesity among adults with type 2 diabetes: the National Health and Nutrition Examination Surveys. $J$ Diabetes Complications 24:368-374.

Lim S and Eckel RH (2014) Pharmacological treatment and therapeutic perspectives of metabolic syndrome. Rev Endocr Metab Disord 15:329-341.

Livak KJ and Schmittgen TD (2001) Analysis of relative gene expression data using real-time quantitative PCR and the 2(-delta delta c(t)) method. Methods 25: $402-408$

Lü J-M, Nurko J, Weakley SM, Jiang J, Kougias P, Lin PH, Yao Q, and Chen C (2010) Molecular mechanisms and clinical applications of nordihydroguaiaretic acid (NDGA) and its derivatives: an update. Med Sci Monit 16:RA93-RA100.

Malik VS, Willett WC, and Hu FB (2013) Global obesity: trends, risk factors and policy implications. Nat Rev Endocrinol 9:13-27.

Memon RA, Tecott LH, Nonogaki K, Beigneux A, Moser AH, Grunfeld C, and Feingold KR (2000) Up-regulation of peroxisome proliferator-activated receptors $($ PPAR- $\alpha$ ) and PPAR- $\gamma$ messenger ribonucleic acid expression in the liver in murine obesity: troglitazone induces expression of PPAR- $\gamma$-responsive adipose tissue-specific genes in the liver of obese diabetic mice. Endocrinology 141 4021-4031

NCD Risk Factor Collaboration (2016) Trends in adult body-mass index in 200 countries from 1975 to 2014: a pooled analysis of 1698 population-based measurement studies with $19 \cdot 2$ million participants. Lancet 387:1377-1396.

Ng M, Fleming T, Robinson M, Thomson B, Graetz N, Margono C, Mullany EC, Biryukov S, Abbafati C, Abera SF, et al. (2014) Global, regional, and national prevalence of overweight and obesity in children and adults during 1980-2013: a systematic analysis for the Global Burden of Disease Study 2013. Lancet 384: 766-781.

Patell R, Dosi R, Joshi H, Sheth S, Shah P, and Jasdanwala S (2014) Non-alcoholic fatty liver disease (NAFLD) in obesity. J Clin Diagn Res 8:62-66.

Pettinelli P and Videla LA (2011) Up-regulation of PPAR- $\gamma$ mRNA expression in the liver of obese patients: an additional reinforcing lipogenic mechanism to SREBP-1c induction. J Clin Endocrinol Metab 96:1424-1430.

Popkin BM and Hawkes C (2016) Sweetening of the global diet, particularly beverages: patterns, trends, and policy responses. Lancet Diabetes Endocrinol 4 174-186.

Rakhshandehroo M, Knoch B, Müller M, and Kersten S (2010) Peroxisome proliferator-activated receptor alpha target genes. PPAR Res 2010:612089.

Rogue A, Spire C, Brun M, Claude N, and Guillouzo A (2010) Gene expression changes induced by PPAR gamma agonists in animal and human liver. PPAR Res 2010:325183.

Seidell JC and Halberstadt J (2016) Obesity: the obesity epidemic in the USA - no end in sight? Nat Rev Endocrinol 12:499-500.

Smith BW and Adams LA (2011) Nonalcoholic fatty liver disease and diabetes mellitus: pathogenesis and treatment. Nat Rev Endocrinol 7:456-465.

Swislocki AL, Siegel D, and Jialal I (2012) Pharmacotherapy for the metabolic syndrome. Curr Vasc Pharmacol 10:187-205

Tanaka Y, Aleksunes LM, Yeager RL, Gyamfi MA, Esterly N, Guo GL, and Klaassen CD (2008) NF-E2-related factor 2 inhibits lipid accumulation and oxidative stress in mice fed a high-fat diet. J Pharmacol Exp Ther 325:655-664. 
Tetri LH, Basaranoglu M, Brunt EM, Yerian LM, and Neuschwander-Tetri BA (2008) Severe NAFLD with hepatic necroinflammatory changes in mice fed trans fats and a high-fructose corn syrup equivalent. Am J Physiol Gastrointest Liver Physiol 295 : G987-G995.

Wadosky KM and Willis MS (2012) The story so far: post-translational regulation of peroxisome proliferator-activated receptors by ubiquitination and SUMOylation. Am J Physiol Heart Circ Physiol 302:H515-H526.

Waller CW and Gisvold O (1945) A phytochemical investigation of Larrea divaricate Cav. J Am Pharm Assoc 34:78-81.

Zalesin KC, Franklin BA, Miller WM, Peterson ED, and McCullough PA (2011) Impact of obesity on cardiovascular disease. Med Clin North Am 95:919-937.

Zhang H, Li Y, Hu J, Shen W-J, Singh M, Hou X, Bittner A, Bittner S, Cortez Y, Tabassum J, et al. (2015) Effect of Creosote bush-derived NDGA on expression of genes involved in lipid metabolism in liver of high-fructose fed rats: relevance to NDGA amelioration of hypertriglyceridemia and hepatic steatosis. PLoS One 10 e0138203.
Zhang H, Shen WJ, Cortez Y, Kraemer FB, and Azhar S (2013) Nordihydroguaiaretic acid improves metabolic dysregulation and aberrant hepatic lipid metabolism in mice by both PPAR $\alpha$-dependent and -independent pathways. Am J Physiol Gastrointest Liver Physiol 304:G72-G86.

Zhang H, Shen W-J, Li Y, Bittner A, Bittner S, Tabassum J, Cortez YF, Kraemer FB, and Azhar S (2016) Microarray analysis of gene expression in liver, adipose tissue and skeletal muscle in response to chronic dietary administration of NDGA to highfructose fed dyslipidemic rats. Nutr Metab (Lond) 13:63.

Address correspondence to: Dr. Wen-Jun Shen, Division of Endocrinology, Gerontology, and Metabolism, Stanford University, 3801 Miranda Avenue, Building 4, Room C302, Palo Alto, CA 94304: E-mail: wenjun@stanford.edu; Dr. Salman Azhar, GRECC-182B. VA Palo Alto Health Care System, 3801 Miranda Avenue, Palo Alto, CA 94304: E-mail: salman.azhar@va.gov 